\title{
Suction/Injection Effects on the Swirling Flow of a Reiner-Rivlin Fluid near a Rough Surface
}

\author{
Bikash Sahoo, ${ }^{1}$ Sébastien Poncet, ${ }^{2,3}$ and Fotini Labropulu ${ }^{4}$ \\ ${ }^{1}$ Department of Mathematics, National Institute of Technology Rourkela, Rourkela 769008, India \\ ${ }^{2}$ Faculté de Génie, Université de Sherbrooke, Sherbrooke, QC, Canada J1K $2 R 1$ \\ ${ }^{3}$ Aix-Marseille Université, CNRS, École Centrale, Laboratoire M2P2 UMR 7340, 13451 Marseille, France \\ ${ }^{4}$ Department of Mathematics, Luther College, University of Regina, Regina, SK, Canada S4S 0A2
}

Correspondence should be addressed to Sébastien Poncet; sebastien.poncet@univ-amu.fr

Received 25 August 2014; Accepted 16 December 2014

Academic Editor: Robert M. Kerr

Copyright (C) 2015 Bikash Sahoo et al. This is an open access article distributed under the Creative Commons Attribution License, which permits unrestricted use, distribution, and reproduction in any medium, provided the original work is properly cited.

\begin{abstract}
The similarity equations for the Bödewadt flow of a non-Newtonian Reiner-Rivlin fluid, subject to uniform suction/injection, are solved numerically. The conventional no-slip boundary conditions are replaced by corresponding partial slip boundary conditions, owing to the roughness of the infinite stationary disk. The combined effects of surface slip $(\lambda)$, suction/injection velocity ( $W$ ), and cross-viscous parameter $(L)$ on the momentum boundary layer are studied in detail. It is interesting to find that suction dominates the oscillations in the velocity profiles and decreases the boundary layer thickness significantly. On the other hand, injection has opposite effects on the velocity profiles and the boundary layer thickness.
\end{abstract}

\section{Introduction}

The problem of Newtonian and non-Newtonian swirling flows near a rotating or stationary disk has occupied a central position in the field of fluid mechanics due firstly to the fact that similarity solutions to the Navier-Stokes equations may be found in some idealized infinite configurations and secondly to its industrial and technical applications in rotating machinery (centrifugal pumps, turbines, or computer storage devices), chemical engineering (spinning disk reactors, crystal growth processes, or rheometers), or oceanography among other things.

Recently, Sahoo [1] and Sahoo and Poncet [2] have obtained numerical solution to similarity equations arising due to steady revolving flow (known as Bödewadt flow [3]) of a non-Newtonian Reiner-Rivlin fluid near an infinite rough stationary disk. In this short note, the flow problem studied by Sahoo and Poncet [2] has been reconsidered, including uniform suction/injection at the surface of the stationary disk. Knowledge of the flow structure close to a porous disk is of practical significance with regard to problems of lubrication of porous bearings or gaseous diffusion among other things. Suction or injection at the surface of a porous disk is also commonly used in chemical engineering to increase the electrochemical reaction time during electrolytic processes [4] or for control purpose as, under given conditions, it delays the transition to turbulence [5]. There are only few attempts in the literature to consider suction/injection at the disk surface. Kelson and Desseaux [6] revisited the von Kármán flow problem over a rotating disk including mass transfer through the disk. Attia [7] extended their work by considering the unsteady flow over an infinite rotating disk with uniform suction and injection and heat transfer effects. Ashraf et al. [8] considered the flow of a micropolar fluid between two stationary disks with constant injection velocity at the surface of one disk. Domairry and Aziz [9] investigated by a homotopy perturbation method the MHD flow between two parallel stationary disks with suction or injection through one of the two disks.

None of these previous works considered the Bödewadt flow of a non-Newtonian fluid over a stationary rough disk with mass transfer through it. This paper is then an endeavour to fill this gap. A second-order finite difference method has been adopted here to solve the resulting system of fully 
coupled and highly nonlinear similarity equations arising due to Reiner-Rivlin swirling flow over an infinite stationary porous disk. The objective is to check if suction or injection is an effective way to reduce the chances of separation of the boundary layer.

\section{Formulation of the Problem}

One considers an incompressible non-Newtonian ReinerRivlin fluid, whose constitutive equation is given by

$$
\mathbf{T}=-p \mathbf{I}+\phi_{1} \mathbf{D}+\phi_{2} \mathbf{D}^{2}
$$

where $\mathbf{D}=(1 / 2)\left[\nabla \mathbf{v}+(\nabla \mathbf{v})^{T}\right][10]$. The response functions $\phi_{1}$ and $\phi_{2}$ are functions of the scalar invariants $(\operatorname{tr} \mathbf{D})^{2}, \operatorname{tr}\left(\mathbf{D}^{2}\right)$, and $\operatorname{det} \mathbf{D}$ [11]. The fluid occupies the space $z>0$ over an infinite stationary disk, which coincides with $z=0$. The motion is due to the rotation of the fluid like a rigid body with constant angular velocity $\Omega$ at large distance from the stationary disk. Let $\mathbf{v}=(u, v, w)$ be the fluid velocity vector in a $(r, \theta, z)$ stationary reference frame (see [2] for a schematic view of the flow configuration). Using the von Kármán transformations [12],

$$
\begin{gathered}
u=r \Omega F(\zeta), \quad v=r \Omega G(\zeta), \quad w=\sqrt{\Omega \nu} H(\zeta), \\
z=\sqrt{\frac{\nu}{\Omega} \zeta, \quad \frac{p}{\rho}=-\nu \Omega P(\zeta)+\frac{1}{2} \Omega^{2} r^{2}}
\end{gathered}
$$

where $v$ is the fluid kinematic viscosity. By considering the usual boundary layer approximations, the equations of continuity and motion take the following forms $[1,2]$ :

$$
\begin{gathered}
\frac{d H}{d \zeta}+2 F=0 \\
\frac{d^{2} F}{d \zeta^{2}}-H \frac{d F}{d \zeta}-F^{2}+G^{2} \\
-\frac{1}{2} L\left[\left(\frac{d F}{d \zeta}\right)^{2}-3\left(\frac{d G}{d \zeta}\right)^{2}-2 F \frac{d^{2} F}{d \zeta^{2}}\right]=1 \\
\frac{d^{2} G}{d \zeta^{2}}-H \frac{d G}{d \zeta}-2 F G+L\left(\frac{d F}{d \zeta} \frac{d G}{d \zeta}+F \frac{d^{2} G}{d \zeta^{2}}\right)=0 \\
\frac{d^{2} H}{d \zeta^{2}}-H \frac{d H}{d \zeta}-\frac{7}{2} L \frac{d H}{d \zeta} \frac{d^{2} H}{d \zeta^{2}}+\frac{d P}{d \zeta}=0
\end{gathered}
$$

where $L=\phi_{2} \Omega / \phi_{1}$ corresponds to the non-Newtonian crossviscous parameter. The above system of equations has to be solved subject to following partial slip boundary conditions:

$$
\begin{gathered}
F(0)=\lambda\left[F^{\prime}(0)-L F(0) F^{\prime}(0)\right], \\
G(0)=\eta\left[G^{\prime}(0)-2 L F(0) G^{\prime}(0)\right], \\
H(0)=W \\
F(\infty) \longrightarrow 0, \quad G(\infty) \longrightarrow 1,
\end{gathered}
$$

where $\lambda$ and $\eta$ are nondimensional slip coefficients and $W=$ $W_{0} / \sqrt{\Omega \nu}$ is the uniform suction $(W<0)$ or injection $(W>0)$ velocity.

The expression of the nondimensional moment coefficient $C_{m}$ is given by

$$
C_{m}=\frac{-\pi\left[G^{\prime}(0)-2 L F(0) G^{\prime}(0)\right]}{\sqrt{\mathbb{R}}},
$$

where $\mathbb{R}=R^{2} \Omega / \nu$ is the Reynolds number based on the disk radius and the fluid velocity far from the disk surface. $C_{m}$ represents the torque required to maintain the disk at rest.

\section{Numerical Solution of the Problem}

In this section, we will present briefly the finite difference method that has been used to solve the system of coupled, nonlinear equations (3)-(5) subject to slip boundary conditions (7). It is customary to mention that similar scheme has been used by Sahoo et al. [13] to solve the Bödewadt flow problem for a viscous fluid with Navier's slip boundary conditions. In this problem, as $H_{0} \neq 0$, a slightly modified scheme has been used in order to get diagonally dominant matrix while using generalized Gauss-Seidel method. The semi-infinite integration domain $[0, \infty)$ is replaced by a finite domain $\left[0, \zeta_{\infty}\right)$. In practice, $\zeta_{\infty}$ should be chosen to be sufficiently large so that the numerical solution closely approximates the terminal boundary conditions and takes into account the asymptotical behavior far from the disk (see Appendix 1 in [14] for the asymptotical behavior of the solutions for large $\zeta$ ). One approximates the functions and their derivatives by their finite difference counterparts and eventually solves a sequence of linear systems as explained below.

(1) One first solves

$$
\begin{aligned}
& {\left[1+L F^{(k)}\right] F^{\prime \prime}-H^{(k)} F^{\prime}} \\
& \quad=\left(F^{(k)}\right)^{2}-\left(G^{(k)}\right)^{2}+\frac{1}{2} L\left[\left(F^{\prime(k)}\right)^{2}-3\left(G^{\prime(k)}\right)^{2}\right]+1
\end{aligned}
$$

using mixed boundary conditions (7) and calls the solution of (9) as $\widetilde{F}^{(k+1)}$, with $k$ being the iteration index. To obtain convergence, one defines $F^{(k+1)}$ by the smoothing formula:

$$
F^{(k+1)}=\alpha_{1} \widetilde{F}^{(k+1)}+\left(1-\alpha_{1}\right) \widetilde{F}^{(k)}, \quad 0 \leq \alpha_{1} \leq 1 .
$$

(2) Then, one solves

$$
\left[1+L F^{(k+1)}\right] G^{\prime \prime}+\left[L F^{(k+1)}-H^{(k)}\right] G^{\prime}=2 F^{(k+1)} G^{(k)}
$$

using derivative boundary conditions (7) and calls the solution of (11) as $\widetilde{G}^{(k+1)}$. To obtain convergence, one defines $G^{(k+1)}$ by the following smoothing formula:

$$
G^{(k+1)}=\alpha_{2} \widetilde{G}^{(k+1)}+\left(1-\alpha_{2}\right) \widetilde{G}^{(k)}, \quad 0 \leq \alpha_{2} \leq 1 .
$$


(3) In this step, one solves

$$
H^{\prime}=-2 F^{(k+1)}
$$

and calls the solution as $\widetilde{H}^{(k+1)}$. To obtain convergence, one defines $H^{(k+1)}$ by the smoothing formula:

$$
H^{(k+1)}=\alpha_{3} \widetilde{H}^{(k+1)}+\left(1-\alpha_{3}\right) \widetilde{H}^{(k)}, \quad 0 \leq \alpha_{3} \leq 1 .
$$

(4) The iterations start with suitable initial guesses $F^{(0)}, G^{(0)}, H^{(0)}, F^{\prime(0)}$, and $G^{(0)}$, borrowed from the work by Sahoo and Poncet [2]. If $F^{(k+1)}, F^{(k)}$, $G^{(k+1)}, G^{(k)}$, and $H^{(k+1)}, H^{(k)}$ are close enough to each other, iterations are stopped; otherwise one sets $k=$ $k+1$ and goes to step (1).

In order to solve the above system of equations by finite difference method, a uniform grid in $0 \leq \zeta \leq \zeta_{\infty}$ is introduced by dividing it into $n$ equal parts with a mesh size $h$ equal to 0.01 . One approximates the derivatives by their finite difference counterparts using second-order schemes as follows:

$$
\begin{aligned}
F^{\prime}\left(\zeta_{i}\right)= & \frac{F_{i+1}-F_{i-1}}{2 h}, \\
F^{\prime \prime}\left(\zeta_{i}\right)= & \frac{F_{i+1}-2 F_{i}+F_{i-1}}{h^{2}}, \\
& i=1,2, \ldots, n-1 .
\end{aligned}
$$

In order to obtain a diagonally dominant linear algebraic system for (9) and (11), $F^{\prime}$ and $G^{\prime}$ are discretized by backward difference approximations as $H_{i}^{(k+1)}>0$ for Bödewadt flow. Finally, (13) is discretized by the central difference approximation. The above algebraic system of equations is solved by generalized Gauss-Seidel method [15]. The convergence of the generalized Gauss-Seidel method for the above diagonally dominant system of equations is quite fast. About 19-21 iterations are necessary to achieve an accuracy of $10^{-6}$. The Fortran 90 code was compiled and run using one of the NIT Rourkela high-end Linux servers. The typical time per iteration for a given mesh distribution $\left(\zeta_{\infty}=20\right.$ and $\left.h=0.01\right)$ and $L=\lambda=1$ is 32.9 seconds for $W=0$ and increases up to 45.4 seconds for $W=1$.

\section{Results and Discussions}

The effects of slip $(\lambda)$ and cross-viscous parameter $(L)$ on the momentum boundary layer have been already precisely discussed in [2]. This short communication focuses only on the effects of the suction/injection velocity on the momentum boundary layer for fixed values of $L=1$ and $\lambda=\eta=1$.

The velocity profiles for the Bödewadt problem exhibit oscillations unlike von Kármán flow. The oscillations occurring in the boundary layer when the fluid rotates near a stationary disk can be explained in the following manner. The radial inflow, induced in the vicinity of the stationary disk, tends to conserve angular momentum and thus to increase

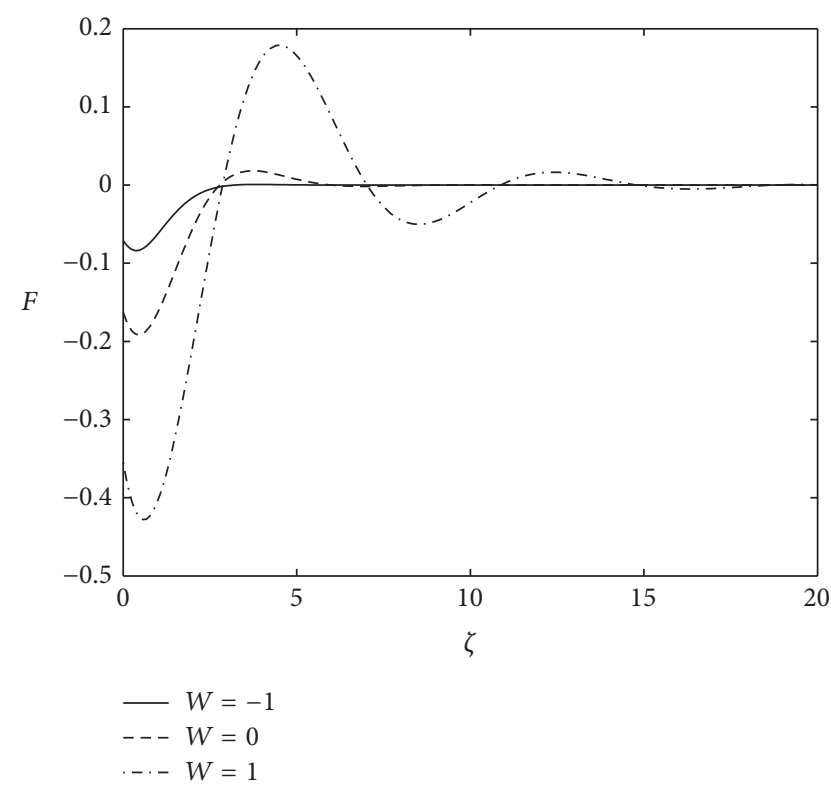

FIGURE 1: Variation of $F$ with $W$.

the tangential velocity when the local radius is decreased. For an overshoot, radial convection of the angular momentum near the disk must be strong enough to more than balance the dissipation of angular momentum caused by the wall shear. This inward radial convection of surplus angular momentum is possible as long as the distribution of circulation in the outer flow increases with increasing radius. A local overshoot in the tangential velocity increases the centrifugal force locally, which tends to induce a radial outflow. This radial outflow convects an angular momentum defect to force an undershoot in the tangential velocity profile, and the above process is repeated to yield oscillatory approach to infinity. It is interesting to observe that the oscillations in the three velocity components reduce as $W$ changes its sign from positive (injection) to negative (suction), as seen in Figures 1 to 3 . The boundary layer thickness decreases significantly when suction is applied. In fact, there is no oscillation in the velocity profiles for $W=-1$. From Figure 1 , it is clear that the intensity of the back flow near the disk surface decreases as $W$ changes sign from positive to negative. Suction decreases the amplitude of the oscillations, whereas injection enhances them. To summarize, suction has a stabilizing effect on the velocity profiles, while injection destabilizes the flow. The influence of $W$ on the components $F$ and $G$ confirms the previous results of Attia [7], in the case of a rotating disk in a porous medium. On the contrary, the effect of $W$ on $H$ has an opposite behavior. The axial velocity component $H$ is relatively constant whatever the distance from the disk is when suction is applied. For future comparisons, the variations of $H_{\infty}, F(0)$, and $G(0)$ for different combinations of the flow parameters are provided in Table 1 . The velocity gradients close to the disk are more interesting. Suction tends to diminish them, which means that the shear stresses may be reduced by decreasing $W$. From Figure 2, it can be seen that the boundary layer thickness is also reduced when $W$ decreases. With injection $W=1$, the fluid is pushed towards 


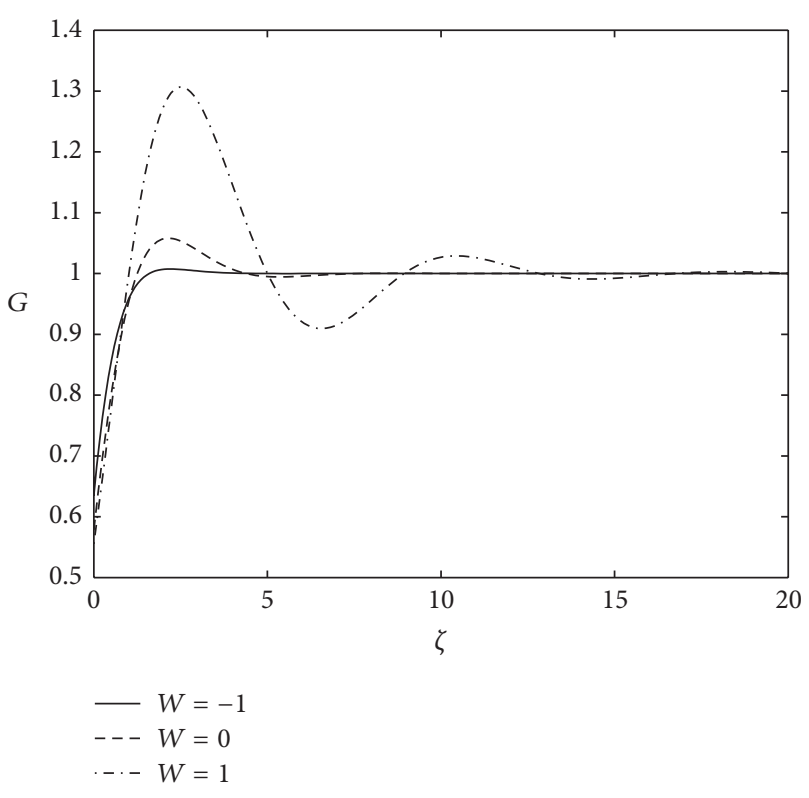

FIGURE 2: Variation of $G$ with $W$.

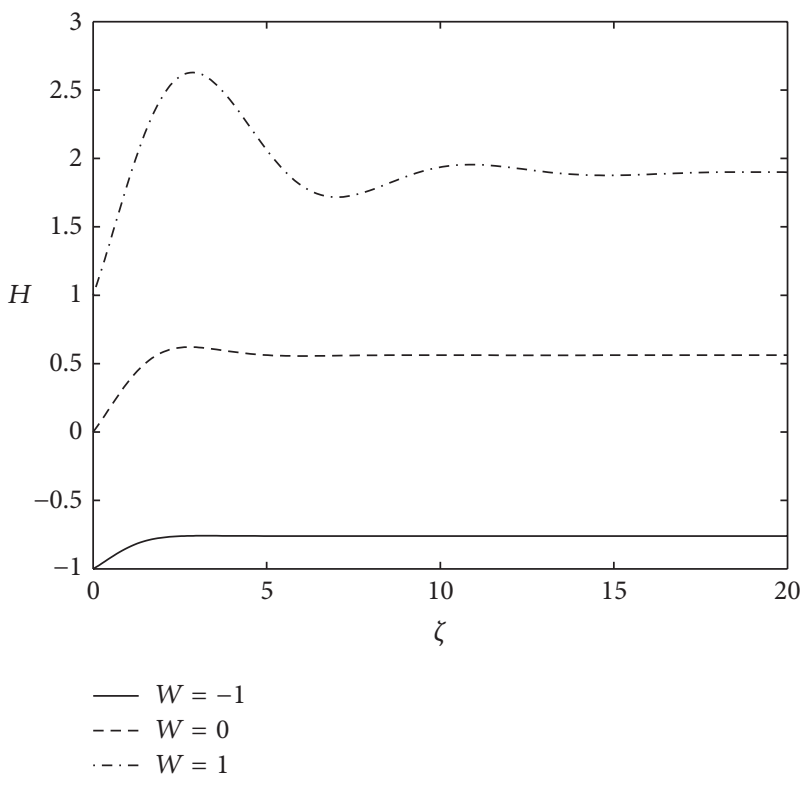

FIgURE 3: Variation of $H$ with $W$.

a larger distance from the disk, which induces a thickening of the boundary layer. The injection velocity $W$ may be then used to adjust both the shear stress and the boundary layer thickness (and as a consequence the velocity profiles) in given engineering applications.

Finally, Figure 4 shows the variation of the moment coefficient $C_{m}$ with the suction/injection velocity $W$ for three different sets of values of $L$ and $\lambda$. Note that the Reynolds number has been fixed to $\mathbb{R}=1$. The moment coefficient $C_{m}$ remains negative for all values of $W$. It is interesting to observe that $W$ has a significant effect on the moment coefficient for a viscous $(L=0)$ Bödewadt flow with no-slip
TABLE 1: Variations of $H_{\infty}, F(0)$, and $G(0)$ with different flow parameters.

\begin{tabular}{lccccc}
\hline$L$ & $\lambda(=\eta)$ & $W$ & $H_{\infty}$ & $F(0)$ & $G(0)$ \\
\hline 0.0 & & & 2.070589 & -0.393258 & 0.510032 \\
1.0 & 1.0 & 1.0 & 1.899414 & -0.261875 & 0.409946 \\
2.0 & & & 1.800522 & -0.195825 & 0.345059 \\
\hline \multirow{3}{*}{1.0} & 1.0 & & 1.899414 & -0.261875 & 0.409946 \\
& 2.0 & 1.0 & 1.522734 & -0.091550 & 0.311701 \\
& 3.0 & & 1.372901 & -0.048644 & 0.239897 \\
\hline \multirow{3}{*}{1.0} & & -1.0 & -0.760271 & -0.066407 & 0.593022 \\
& 1.0 & 0.0 & 0.561328 & -0.140015 & 0.497071 \\
& & 1.0 & 1.899414 & -0.261876 & 0.409946 \\
\hline
\end{tabular}

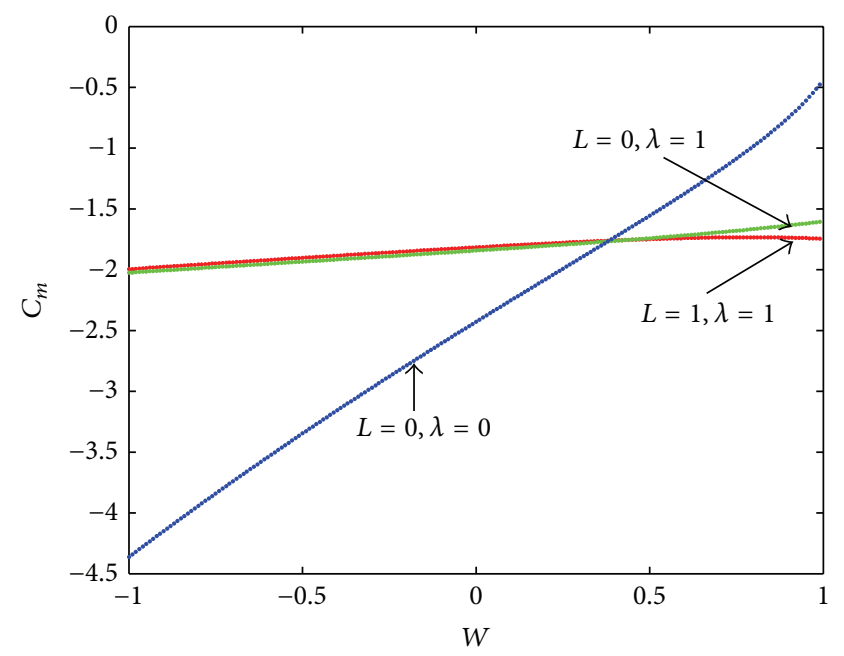

Figure 4: Variation of $C_{m}$ with $W$.

$(\lambda=0)$ boundary conditions. In this case, the torque required to maintain the disk at rest is high when there is suction at the disk surface and it decreases in magnitude as $W$ changes its sign from negative to positive. The rate of decrease of $C_{m}$ as $W$ changes sign from negative to positive for the other two cases $(L=0, \lambda=1 ; L=1, \lambda=1)$ is not significant. It is also observed that, for the Reiner-Rivlin fluid $(L \neq 0), C_{m}$ decreases in magnitude when $W$ varies in the range $[-1,1]$ up to a critical value $W^{*}$. For $L=1$ and $\lambda=1, W^{*} \simeq 0.75$.

\section{Conclusions}

In this short communication, we have investigated the effects of suction and injection on the momentum boundary layer arising due to the swirling flow of a non-Newtonian ReinerRivlin fluid over an infinite rough stationary disk. A secondorder finite difference method has been adopted to solve the resulting system of fully coupled and highly nonlinear similarity equations. It is observed that suction suppresses the oscillations in the velocity profiles, whereas injection enhances it. The boundary layer thickness decreases as the suction increases. Injection has an opposite effect on it. 


\section{Conflict of Interests}

The authors declare that there is no conflict of interests regarding the publication of this paper.

\section{References}

[1] B. Sahoo, "Effects of slip on steady Bödewadt flow and heat transfer of an electrically conducting non-Newtonian fluid," Communications in Nonlinear Science and Numerical Simulation, vol. 16, no. 11, pp. 4284-4295, 2011.

[2] B. Sahoo and S. Poncet, "Effects of slip on steady Bödewadt flow of a non-Newtonian fluid," Communications in Nonlinear Science and Numerical Simulation, vol. 17, no. 11, pp. 4181-4191, 2012.

[3] U. T. Bödewadt, "Die Drehströmung über festem Grunde," ZAMM-Zeitschrift für Angewandte Mathematik und Mechanik, vol. 20, no. 5, pp. 241-253, 1940.

[4] R. T. Bonnecaze, N. Mano, B. Nam, and A. Heller, "On the behavior of the porous rotating disk electrode," Journal of the Electrochemical Society, vol. 154, no. 2, pp. F44-F47, 2007.

[5] R. J. Lingwood, "On the effects of suction and injection on the absolute instability of the rotating-disk boundary layer," Physics of Fluids, vol. 9, no. 5, pp. 1317-1328, 1997.

[6] N. Kelson and A. Desseaux, "Note on porous rotating disk flow," ANZIAM Journal, vol. 42, pp. C837-C855, 2000.

[7] H. A. Attia, "On the effectiveness of uniform suction and injection on unsteady rotating disk flow in porous medium with heat transfer," Computational Materials Science, vol. 38, no. 2, pp. 240-244, 2006.

[8] M. Ashraf, M. A. Kamal, and K. S. Syed, "Numerical simulation of flow of a micropolar fluid between a porous disk and a nonporous disk," Applied Mathematical Modelling, vol. 33, no. 4, pp. 1933-1943, 2009.

[9] G. Domairry and A. Aziz, "Approximate analysis of MHD dqueeze flow between two parallel disks with suction or injection by homotopy perturbation method," Mathematical Problems in Engineering, vol. 2009, Article ID 603916, 19 pages, 2009.

[10] W. R. Schowalter, Mechanics of Non-Newtonian Fluids, Pergamon Press, Oxford, UK, 1978.

[11] D. R. Smith, An Introduction to Continuum Mechanics-after Truesdell and Noll, vol. 22 of Solid Mechanics and its Applications, Kluwer Academic, Dordrecht, The Netherlands, 1993.

[12] T. von Kármán, "Über laminare und turbulente Reibung," Zeitschrift für Angewandte Mathematik und Mechanik, vol. 1, no. 4, pp. 233-252, 1921.

[13] B. Sahoo, S. Abbasbandy, and S. Poncet, "A brief note on the computation of the Bödewadt flow with Navier slip boundary conditions," Computers and Fluids, vol. 90, pp. 133-137, 2014.

[14] A. I. van de Vooren, E. F. F. Botta, and J. Stout, "The boundary layer on a disk at rest in a rotating fluid," Quarterly Journal of Mechanics and Applied Mathematics, vol. 40, no. 1, pp. 15-32, 1987.

[15] D. K. Salkuyeh, "Generalized Jacobi and Gauss-Seidel methods for solving linear system of equations," Numerical Mathematics A: Journal of Chinese Universities, vol. 16, no. 2, pp. 164-170, 2007. 

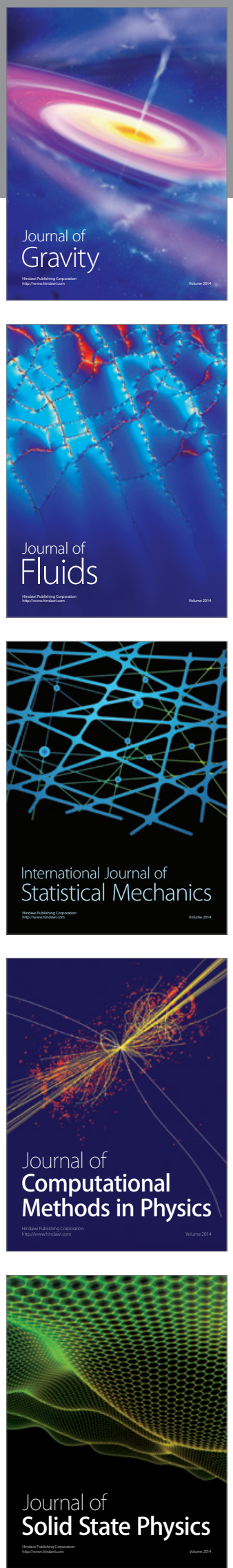

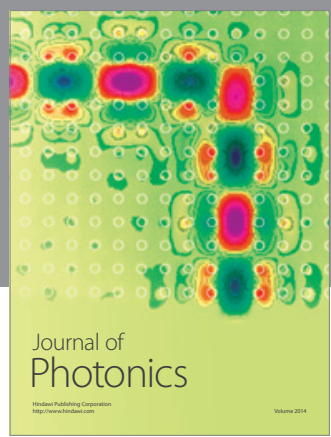

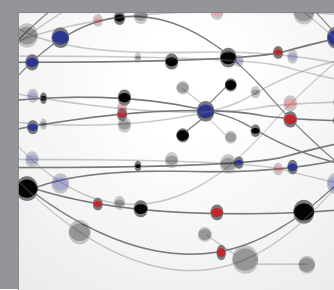

The Scientific World Journal

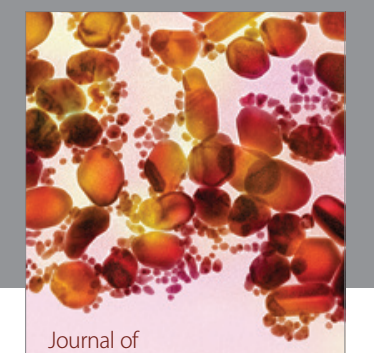

Soft Matter
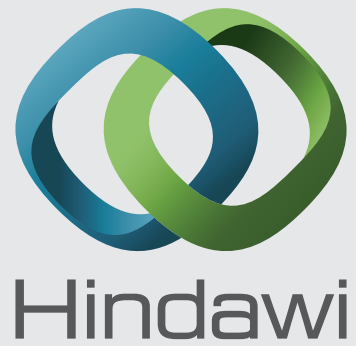

Submit your manuscripts at

http://www.hindawi.com
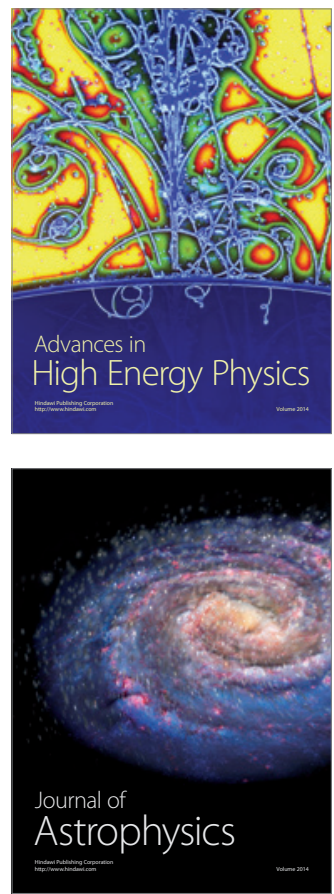
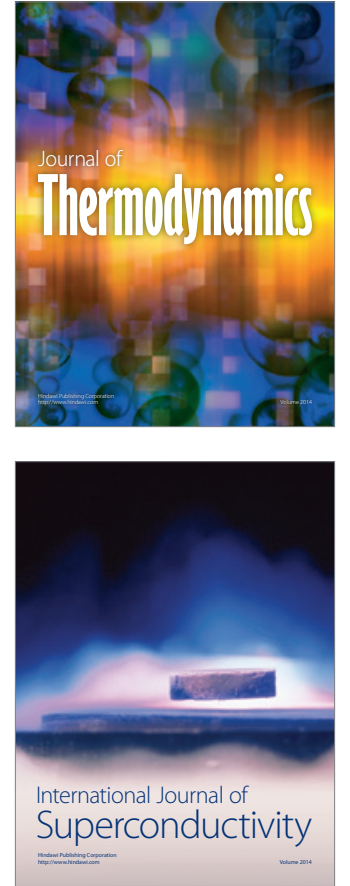
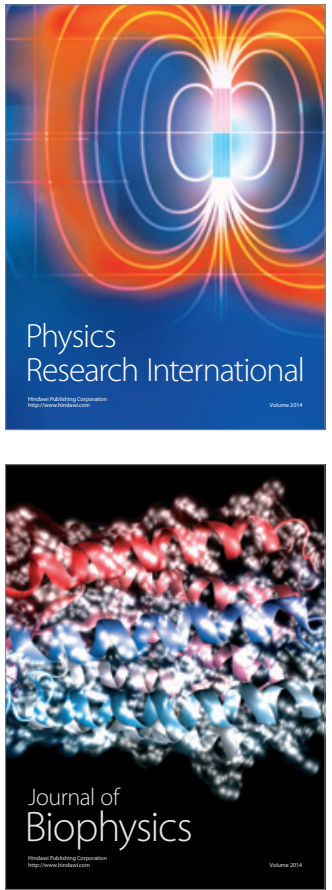
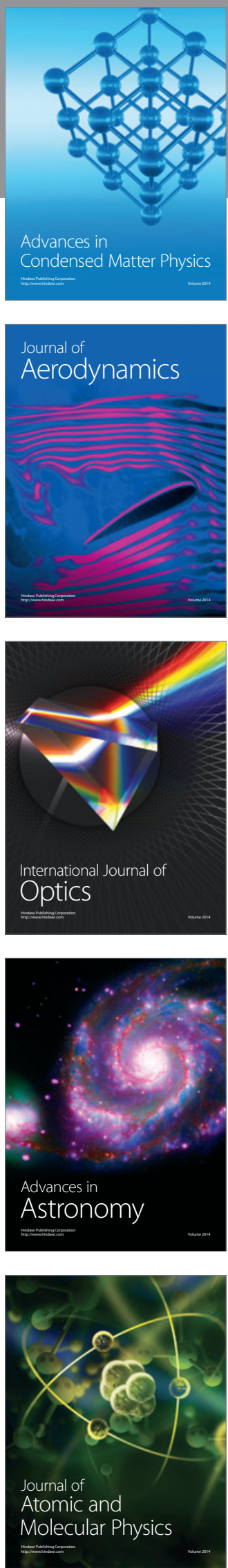